ARTÍCULO ORIGINAL

\title{
Gestión de la investigación en Honduras: perspectiva desde la Revista Médica Hondureña
}

\author{
Research management in Honduras: perspective through the Revista Médica Hondureña
}

Carlos J. Fajardo, ${ }^{1}$ Jackeline Alger. $^{2}$

${ }^{1}$ Médico General, Candidato al grado de Maestría en Epidemiología, Facultad de Ciencias Médicas (FCM), Universidad Nacional Autónoma de Honduras (UNAH); Centro de Cáncer Emma Romero de Callejas, Tegucigalpa.

${ }^{2}$ Médica, Doctorado (PhD) en Parasitología, Unidad de Investigación Científica; FCM, UNAH; Servicio de Parasitología, Departamento de Laboratorio Clínico, Hospital Escuela; Tegucigalpa.

RESUMEN. Antecedentes: La Revista Médica Hondureña $(\mathrm{RMH})$ registra información sobre asistencia clínica, salud pública, educación e investigación. La gestión del conocimiento para la investigación en salud organiza los flujos de información y propicia la generación, apropiación, intercambio y uso de conocimientos necesarios para el incremento de la eficacia de la investigación. Objetivo: Describir la gestión de la investigación en Honduras a través de las publicaciones en la RMH (19302020). Métodos: Revisión bibliométrica; se realizó búsqueda bajo el término investigación (http://www.bvs.hn/RMH/html5/\#gsc. $\underline{t a b}=0)$. Se seleccionaron los artículos de acuerdo a definición operativa de gestión de la investigación en base a los objetivos de la Política de Investigación para la Salud (OPS/OMS); se clasificaron por año de publicación, tipo de artículo, tema, ámbito. Resultados: Se identificaron 24 artículos (1989-2020); según tipo de artículo: Opinión 12, Editorial 5, Original 3, Especial 2, Revisión bibliográfica 1, Historia 1; según área temática general, se abordaron uno o más temas, principalmente: Generación de investigaciones 15, Competencia del talento humano 15 , Priorización de la investigación 9, Difusión y utilización de resultados 9, Alianzas estratégicas 8 . En cuanto al ámbito, 17 ámbito nacional, 3 regional, 4 global. Discusión: La RMH refleja avances y desafíos de la investigación para la salud en Honduras. Es necesario promover iniciativas e investigaciones sobre gobernanza, prácticas y estándares y mecanismos de evaluación de la investigación. La creación de un sistema nacional de investigación para la salud contribuiría a promover la generación y uso de la evidencia científica y por consiguiente un mejor sistema de salud pública.

Palabras clave. Investigación, Gestión del conocimiento para la investigación en salud, Política de investigación en salud, Promoción de la investigación.

Recibido: 12-11-2020 Aceptado para publicación 22-12-2020

Dirección para correspondencia: Dr. Carlos J. Fajardo

Correo electrónico: cjfajardo92@gmail.com

Declaración de relaciones y actividades financieras y no financieras y conflictos de interés: ninguno.

DOI: $h$ ttps://doi.org/10.5377/rmh.v88i2.11489

\section{INTRODUCCIÓN}

La Revista Médica Hondureña (RMH), publicada desde 1930, en el presente año conmemora su Noventa Aniversario. ${ }^{1,2}$ Por su antigüedad y por el tipo de información que registra, la revista se ha convertido en un referente nacional para analizar aspectos clave en el campo de la salud en Honduras y percibidos desde Honduras a nivel regional y global. ${ }^{3} \mathrm{~A}$ lo largo de nueve décadas, a través de sus artículos, la revista nos presenta eventos y aspectos evolutivos de la asistencia clínica, salud pública, historia, educación e investigación para la salud en el país. La gestión del conocimiento para la investigación en salud se define como un sistema que organiza los flujos de información, externos e internos, y propicia la generación, apropiación, intercambio y uso de conocimientos necesarios para el incremento de la eficacia de la investigación en ciencia y tecnología en salud (https://bit.ly/38NbHDH).

Es reconocido qu e la investigación para la salud contribuye a reducir las desigualdades, mejorar la salud de la población y acelerar el desarrollo socioeconómico de los países, y es una fuente esencial de soluciones a los problemas de salud para responder con eficiencia, eficacia, y equidad. ${ }^{4} \mathrm{~A}$ partir de la década de los 90 s, comenzó un ascenso en la actividad en investigación en salud a nivel mundial en respuesta al hecho que menos del $10 \%$ de la inversión global se dirigía hacia los padecimientos que causan el $90 \%$ de la carga global de enfermedad. ${ }^{5}$ Sin embargo, este crecimiento en la investigación no ha sido uniforme entre las diversas regiones del planeta. América Latina solamente invierte un $0.6 \%$ de su PIB en investigación y desarrollo, cifra mucho menor al 2-3\% que invierten los países desarrollados. ${ }^{6}$ En Honduras por su parte, este indicador es de $0.01 \% .^{7}$ Para desarrollarse, la investigación de calidad necesita un ambiente con gobernanza eficiente en donde se determinen prioridades, se desarrollen capacidades de investigación (talento humano, inversión e institucionalidad), se establezcan estándares de prácticas de investigación y se trasladen los resultados de investigaciones a políticas en salud. ${ }^{6}$ Por lo tanto, se promueve que los países cuenten con sistemas nacionales de investigación para la salud y se incremente la producción y 
utilización de investigaciones que favorezcan alcanzar metas como la cobertura de salud universal y los objetivos de desarroIlo sostenible (ODS). ${ }^{7,8}$ Recientemente, los países de la región establecieron la Agenda de Salud Sostenible de las Américas 2018-2030 en la cual el Objetivo 7 plantea desarrollar capacidades para la generación, la transferencia y el uso de la evidencia y el conocimiento en materia de salud, promoviendo la investigación, la innovación y el uso de la tecnología. ${ }^{9}$

Honduras no cuenta con un sistema nacional de investigación para la salud, aunque cuenta con algunos componentes del mismo como prioridades de investigación y algunas leyes y regulaciones. ${ }^{10-12}$ Se realizó un análisis bibliométrico con el objetivo de describir la gestión de la investigación en Honduras a través de las publicaciones en la RMH. Esperamos que la información presentada en este artículo contribuya a comprender aspectos de la investigación para la salud en el país y algunas de las necesidades para su gestión efectiva y fortalecimiento.

\section{MATERIALES Y MÉTODOS}

Se realizó un análisis bibliométrico de las publicaciones de la RMH con contenido sobre la gestión de la investigación en Honduras. Se utilizó como referencia el diseño tipo "scoping review" (revisión de alcance o de reconocimiento) por su utilidad para sintetizar evidencia científica (ver detalles en el enlace: https://guides.temple.edu/c.php?g=78618\&p=4156607). Utilizando el término investigación se realizó búsqueda temática en la RMH mediante dos estrategias, una con apoyo del personal de la Biblioteca Médica Nacional y otra directamente en las páginas de la RMH en la Biblioteca Virtual en Salud de Honduras (http://www.bvs.hn/RMH/html5/\#gsc.tab=0).

Se utilizaron los siguientes criterios de inclusión de las publicaciones: artículo publicado desde 1930 en un número de cada volumen de la RMH, sin incluir suplementos, y que en su contenido se refiriera a temas generales relacionados a la gestión de la investigación como ser: 1) Generación de investigaciones, 2) Gobernanza de la investigación, 3) Priorización de la investigación, 4) Competencia del talento humano que participa en investigación, 5) Alianzas estratégicas y la participación del público en la investigación, 6) Prácticas y estándares óptimos para la investigación, 7) Difusión y utilización de los resultados de la investigación, y 8) Mecanismos de evaluación de la investigación. Esta temática general se identificó a partir de los objetivos de la Política de Investigación para la Salud de la Organización Panamericana de la Salud/Organización Mundial de la Salud. ${ }^{4}$ No se incluyeron los artículos sobre ética de la investigación e integridad científica por ser el objeto de otro estudio. ${ }^{13}$ Se realizó un tamizaje inicial por título y resumen cuando estuviera disponible. Posteriormente se realizó una revisión del artículo completo para determinar su inclusión en el estudio.

Una vez seleccionados los artículos con contenido sobre gestión de la investigación, se procedió a clasificarlos por año de publicación, tipo de artículos y tema específico. A un artículo se le asignó más de un tema, dependiendo de su contenido. Además, se clasificaron de acuerdo al abordaje de temas de gestión de la investigación en el ámbito nacional, regional o global. El ámbito nacional incluyó los artículos que abordaron temas locales o nacionales originados por cualquier institución hondureña; ámbito regional aquellos que abordaron estrategias o experiencias cuya iniciativa o temática involucra a países de América Latina; y el ámbito global aquellos que abordaron temáticas de gestión de investigación que surgieron de organismos internacionales e incluyeron a países de varios continentes. Los resultados se presentan como frecuencias de las variables analizadas. Se presenta un diagrama de flujo para describir el proceso de selección y evaluación de los artículos (Figura 1).

\section{RESULTADOS}

A través de ambas estrategias de búsqueda en el periodo 1930 a 2020 se identificó un total de 25 artículos publicados en la RMH, de acuerdo al término investigación. Los artículos correspondieron al período 1967 a 2020. De éstos, en 24 artículos se identificó contenido relacionado a la gestión de la investigación y correspondieron al período 1989 a 2020 (Figura 1).

En el Cuadro 1 se presenta una distribución de los artículos por año y de acuerdo al tipo de artículo, tema específico y área temática general. El tipo de artículo incluyó artículos tipo Opinión 12, Editorial 5, Original 3, Especial 2, Revisión bibliográfica

Figura 1. Diagrama de flujo del proceso de selección y evaluación de artículos publicados en Revista Médica Hondureña, 1930-2020.

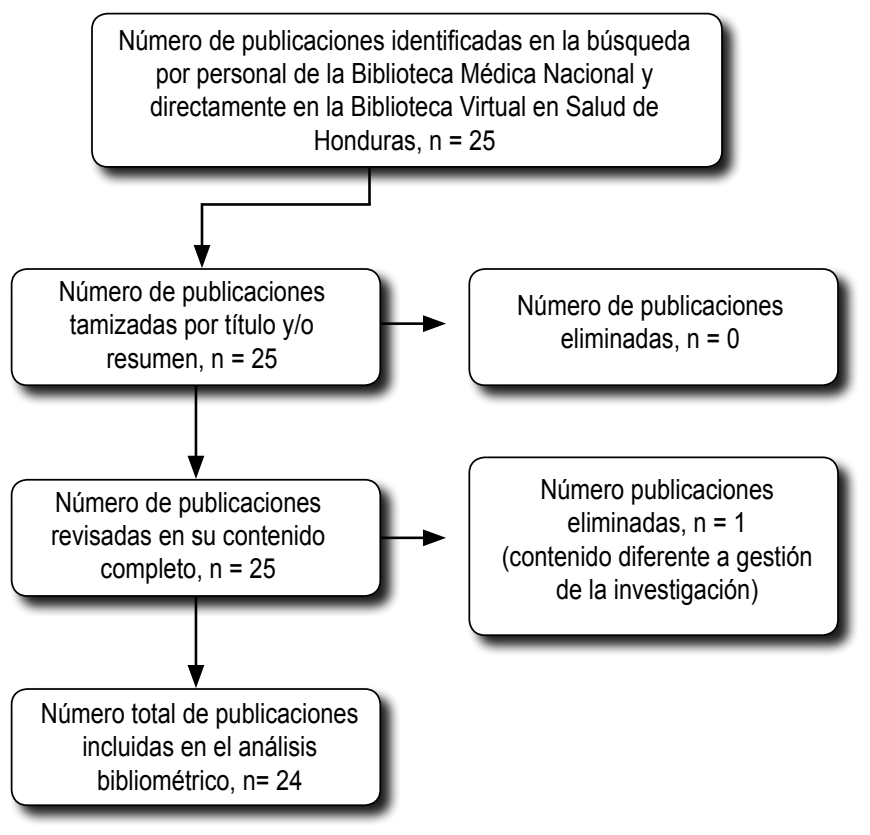

1, Historia de la Medicina 1. De acuerdo al área temática general, los artículos abordaron Generación de investigaciones 15, Competencia del talento humano que participa en investigación 15, Priorización de la investigación 9, Difusión y utilización de los resultados de la investigación 9 , Alianzas estratégicas y la participación del público en la investigación 8, Gobernanza de la investigación 5 , Prácticas y estándares óptimos para la investigación 5 , y Mecanismos de evaluación de la investigación 2 
Cuadro 1. Características de los artículos sobre gestión de la investigación de acuerdo al año, tipo de artículo y tema, Revista Médica Hondureña, $1989-2020, \mathrm{n}=24$.

\begin{tabular}{|c|c|c|c|c|c|}
\hline AÑO & TIPO DE ARTÍCULO & & ÁREA TEMÁTICA GENERAL** & $\begin{array}{l}\text { TEMÁTICA } \\
\text { ESPECÍFICA* }\end{array}$ & $\begin{array}{l}\text { INICIATIVA NACIONAL/ } \\
\text { REGIONAL/ GLOBAL }\end{array}$ \\
\hline \multirow[t]{2}{*}{1989} & Opinión & 1) & ¿Por qué debe, además de enseñar, escribir? ${ }^{15}$ & 4 & Nacional \\
\hline & Opinión & 2) & Investigación como parte de formación profesional ${ }^{16}$ & 4 & Nacional \\
\hline 1991 & Opinión & 3) & Políticas de Investigación en la UNAH y FCM ${ }^{17}$ & $1,2,3,4$ & Nacional \\
\hline 1996 & Editorial & 4) & Políticas de Investigación en las Ciencias Biomédicas ${ }^{31}$ & 2,4 & Nacional \\
\hline 1997 & Original & 5) & Práctica de la Investigación ${ }^{28}$ & 1,4 & Nacional \\
\hline \multirow[t]{3}{*}{2002} & Editorial & 6) & La Revista Médica Hondureña, la investigación biomédica y alianzas ${ }^{27}$ & 1,7 & Nacional \\
\hline & Opinión & 7) & Foro Mundial para la Investigación en Salud ${ }^{19}$ & $1,3,4,5$ & Global \\
\hline & Especial & 8) & $\begin{array}{l}\text { Programa Especial para Investigación y Entrenamiento en Enfermedades } \\
\text { Tropicales }^{18}\end{array}$ & $1,3,4,5$ & Global \\
\hline \multirow[t]{2}{*}{2003} & Historia de la Medicina & 9) & Reseña histórica del Congreso Médico Nacional ${ }^{33}$ & $1,4,7$ & Nacional \\
\hline & Opinión & 10) & XLVI Congreso Médico Nacional Dr. Pablo J. Cambar ${ }^{35}$ & $1,3,6,7$ & Nacional \\
\hline 2006 & Opinión & 11) & 5to Congreso Internacional de Revisión por Pares y Publicación Biomédica²0 & $4,6,7,8$ & Global \\
\hline 2007 & Opinión & 12) & Curso y reunión de editores hondureños. ${ }^{23}$ & $4,5,6,7,8$ & Nacional \\
\hline \multirow[t]{2}{*}{2008} & Opinión & 13) & $\begin{array}{l}\text { 1ra Conferencia Latinoamericana sobre Investigación e Innovación para la } \\
\text { Salud }{ }^{21}\end{array}$ & $1,2,3,5,7$ & Regional \\
\hline & Editorial & 14) & Docencia y investigación en el Instituto Nacional Cardiopulmonar & 3,4 & Nacional \\
\hline \multirow[t]{2}{*}{2010} & Opinión & 15) & Reunión de la Red Inter-regional de Centros de Referencia ${ }^{22}$ & $1,4,5$ & Global \\
\hline & Opinión & 16) & La Asociación Hondureña de Editores Científicos (AHECI) $)^{24}$ & $4,5,7$ & Nacional \\
\hline \multirow[t]{2}{*}{2011} & Editorial & 17) & Vanguardia de la Revista Médica Hondureña ${ }^{25}$ & 1,7 & Nacional \\
\hline & Opinión & 18) & XVI Congreso de los postgrados de Medicina ${ }^{34}$ & $1,3,4$ & Nacional \\
\hline \multirow[t]{2}{*}{2013} & Especial & 19) & HRWeb (Health Research Web) $)^{32}$ & $2,4,5,6$ & Regional \\
\hline & Opinión & 20) & EVIPNet (Evidence-informed Policy Network) ${ }^{42}$ & 2,5 & Regional \\
\hline 2014 & Editorial & 21) & Un nuevo reto en la publicación científica en medicina ${ }^{26}$ & 1 & Nacional \\
\hline 2016 & Original & 22) & Investigación en el posgrado de Ginecología y Obstetricia ${ }^{29}$ & 1,3 & Nacional \\
\hline 2018 & Original & 23) & Características de trabajos de investigación ${ }^{30}$ & $1,3,7$ & Nacional \\
\hline 2019 & Revisión Bibliográfica & 24) & Investigación de la Implementación ${ }^{39}$ & 1,6 & Nacional \\
\hline
\end{tabular}

*Temática específica: superíndices representan las referencias citadas en la sección correspondiente. **Área temática general: 1. Generación de investigación 2. Gobernanza de la investigación 3. Priorización de la Investigación 4. Competencia del talento humano que participa en Investigación 5 . Alianzas estratégicas y la participación del público en la investigación 6 . Prácticas y estándares óptimos para la investigación 7 . Difusión y utilización de los resultados de la investigación 8. Mecanismos de evaluación de la investigación.

(Cuadro 1). En cuanto al ámbito de abordaje de la gestión de la investigación, 17 provienen del ámbito nacional, 3 del regional y 4 del global.

\section{DISCUSIÓN}

La RMH, el referente nacional de la evolución del conocimiento médico y de políticas en salud, muestra a través de sus 90 años de historia el panorama de la investigación en nuestro país. Aunque los resultados no pueden generalizarse, ya que se limitan a una sola fuente, a través de este análisis bibliométrico se refleja que la RMH ha sido y seguirá siendo una plataforma importante para contribuir a impulsar la investigación en nuestro país. Todavía hay un largo camino por recorrer para reconocer a la investigación como un motor de desarrollo y una pieza clave para el fortalecimiento del sistema de salud en Honduras.
El primer artículo publicado que hace mención a la investigación fue en 1967 en un editorial donde destaca la labor de la Facultad de Ciencias Médicas (FCM) de la Universidad Nacional Autónoma de Honduras (UNAH), específicamente del Departamento de Ciencias Fisiológicas. A propósito de la publicación de un trabajo de investigación sobre los efectos metabólicos y antitumorales de una saponina extraída del helecho calaguala (Polypodium leucotomos) se señala "Consideramos que debe ser motivo de orgullo para nuestra Universidad, haber logrado la iniciación de estudio de investigación científica experimental." ${ }^{14}$ Sin duda que esto suponía un futuro brillante para la investigación en ciencias básicas en nuestro país. No obstante, fue hasta 1989 cuando en dos artículos de opinión se retoma la importancia de la investigación clínica; el primero resaltando la importancia de que los cirujanos clínicos y docentes se involucraran en la escritura de artículos científicos $y$ el segundo abordando la necesidad de formar personal 
médico especializado que, teniendo un papel protagónico en la investigación científica, mejoraran la calidad de la salud del pueblo hondureño. ${ }^{15,16}$ En ambas publicaciones se enfatiza que las instituciones para las que laboran los médicos, deben impulsarlos y motivarlos para que realicen investigaciones.

Un hito importante que destaca en este recorrido histórico es la creación en 1988 de la Unidad de Investigación Científica (UIC), UNAH, aprobada por el Consejo Universitario en 1990 con "la función principal de definir y elaborar la política de investigación para la Facultad de Ciencias Médicas y organizar la unidad responsable de promover y coordinar la actividad investigativa en la Facultad."17 Esta compleja tarea se planeaba realizar mediante un proceso de desarrollo de la investigación que tomaba en cuenta las siguientes áreas básicas de trabajo: a) definición de políticas y prioridades; b) capacitación y apoyo técnico a los investigadores; c) promoción, asesoría y monitoreo de proyectos de investigación; y d) documentación y divulgación. ${ }^{17}$ Todo esto para contrarrestar la cultura de investigación existente que se caracterizaba por ser producto de la iniciativa individual del investigador y no de políticas nacionales de investigación.

Entre el 2002 y 2010 , se escribieron 11 artículos describiendo iniciativas y experiencias tanto nacionales, regionales y mundiales cuya relevancia es de interés en el medio investigativo. Una de ellas publicada en 2002 como Programa Especial para Investigación y Entrenamiento en Enfermedades Tropicales - TDR, el cual fue creado con las disposiciones de conducir investigaciones para desarrollar herramientas que ayudaran al control de un grupo definido de enfermedades. ${ }^{18}$ Otra importante iniciativa mundial fue la descripción del evento desarrollado en Ginebra, Suiza: Foro Mundial para la investigación en Salud - Foro 5, cuyos objetivos planteados fueron: "desarrollar metodologías de establecimiento de prioridades que permitan identificar mejor las prioridades en la investigación en salud; segundo, promover el desarrollo de redes que asocien un amplio rango de colaboradores en la búsqueda de soluciones al establecimiento de prioridades de los problemas de salud a través de la investigación; y tercero, realizar la reunión anual, el Foro, para evaluar progresos. ${ }^{199} \mathrm{En} 2006$, se publicó un artículo titulado Quinto Congreso Internacional de Revisión por Pares y Publicación Biomédica desarrollado en Chicago, Illinois, cuyo objetivo principal fue "mejorar la calidad y credibilidad del proceso de revisión por pares o arbitraje y la publicación biomédica y apoyar el avance de la eficiencia, efectividad y equidad de la diseminación de la información biomédica a través del mundo."20 La publicación de estas iniciativas mundiales servían como vitrinas que mostraban las realidades y parámetros utilizados en países de altos ingresos y que permitirían guiar las investigaciones nacionales.

Son visibles también en la década de los 2000s, iniciativas latinoamericanas como por ejemplo la Primera Conferencia Latinoamericana sobre Investigación e Innovación para la Salud, Río de Janeiro, Brasil, en 2008, presentada por un ecléctico equipo de autores de varios países de América que relataron el evento. Este último tenía el propósito de aumentar la contribu- ción de la investigación a la salud y la equidad en América Latina. ${ }^{21}$ Una de las mayores necesidades para el desarrollo de la investigación en la región es la capacitación del talento humano que permita fortalecer las habilidades de los investigadores de países en vías de desarrollo, que posibilite la implementación de sus proyectos y el establecimiento de colaboraciones. Esto fue el tema central de la Reunión de la red inter-regional de centros de referencia para capacitación en cursos de planeación y evaluación efectivas de proyectos de investigación para la saIud, Cali; Colombia, abril 2010.22 También fueron publicadas experiencias nacionales orientadas a la capacitación de editores de revistas médicas hondureñas y el fortalecimiento de la publicación nacional a través de diversas actividades incluyendo la creación de la Asociación Hondureña de Editores Científicos (AHECl). ${ }^{23,24 .}$

Los editoriales han sido un espacio propicio para abordar temas de gestión de la investigación. En 2011 se discute que "la investigación científica es esencial para garantizar la salud de la población con calidad, igualdad, acorde a las características epidemiológicas y socioeconómicas del país", promoviendo la confianza para utilizar y citar los trabajos de investigación científica generados en nuestro país. ${ }^{25}$ Además se destaca en un editorial en 2014 que el idioma español aporta solamente 1\% de la literatura médica, y exhorta de igual forma a los médicos hondureños a disminuir esa brecha mediante la publicación de investigaciones nacionales ${ }^{26}$ Una realidad ampliamente conocida es que en Honduras hay acceso limitado a datos de calidad que indiquen confiablemente cual es la situación de la investigación científica, como es mencionado en un editorial que expone "uno de los principales escollos para sostener la estructura del contenido [de la Revista Médica Hondureña] es el limitado flujo de trabajos originales."${ }^{\prime 27}$ Esta aseveración se ve reflejada en el hecho que este análisis bibliométrico solo identificó tres artículos originales que generan datos inéditos, todos ellos con un diseño observacional. ${ }^{28-30}$ Uno de ellos publicado en 1997 sobre la práctica de la investigación médica en la Facultad de Ciencias Médicas UNAH, cuyos resultados explican la baja calidad y cantidad de publicaciones entre los docentes, incluyendo la necesidad de capacitación en manejo computarizado de datos, diseño y métodos de investigación científica y operativa, redacción científica y técnicas cualitativas de investigación; la falta de financiamiento para realizar investigaciones y la falta de incentivos. ${ }^{28}$ Casi 20 años después, en 2016, un estudio original caracterizó la investigación realizada por el Postgrado de Ginecología y Obstetricia 2012-2015 mostrando que aún persistían los mismos obstáculos para la investigación: estudios hospitalarios predominantemente descriptivos que no cuentan con un dictamen de comité de ética, con recursos limitados y ausencia de asesoría metodológica. ${ }^{29}$

La práctica de investigación científica se correlaciona con el desarrollo general de los países, como se expone en un editorial publicado en 1996, "Una de las diferenciaciones básicas entre países desarrollados y subdesarrollados se basa en la rapidez como dichas sociedades logran realizar investigación e incorporar los conocimientos a dicha sociedad." ${ }^{11}$ Para lograr 
acortar esta brecha en las capacidades en investigación entre países de altos, medianos y bajos ingresos, es necesario contar con un Sistema Nacional de Investigación para la Salud que esté bien organizado y sea una prioridad para el gobierno. El artículo especial publicado en 2013, HRWeb: una herramienta para facilitar una mejor gobernanza de la investigación, presenta el concepto "Health Research Web es una plataforma web interactiva que facilita la recopilación de información esencial que caracteriza los sistemas de investigación en salud." La HRWeb (https://www.healthresearchweb.org/) presenta la posibilidad de convertirse en una comunidad en línea, conformada por personas e instituciones interesadas en desarrollar información sobre investigaciones de alta calidad y un sistema de gestión en un entorno de respeto mutuo. Siendo una herramienta de referencia para aquellos que desean establecer vínculos y desarrollar investigaciones en colaboración, ya sea como investigadores independientes, como parte de organizaciones e incluso sistemas de salud. ${ }^{32}$ Los congresos médicos organizados por el Colegio Médico de Honduras son otra importante plataforma para la gestión, generación y presentación de la investigación científica. Una característica común de todos los artículos que hacen referencia a los congresos médicos del Colegio Médico de Honduras es que el fortalecimiento de la investigación es un pilar fundamental para el mejoramiento de la salud en el país, ya que la generación de evidencia propia incrementa el conocimiento científico y técnico que permite la toma de decisiones que vayan en beneficio de la población hondureña. ${ }^{33-35}$

Es necesario que la investigación para la salud se lleve a la acción, es decir que la evidencia generada se convierta en políticas públicas. Para esto es evidente la necesidad de contar con un sistema nacional de investigación que establezca las prioridades locales de investigación y además facilite los recursos necesarios para realizar investigaciones de calidad. En 2009 , en un informe especial se realizó una revisión de los sistemas nacionales de investigación para la salud en 14 países de América Latina que participaron en la Primera conferencia Latinoamericana sobre Investigación e Innovación para la salud. ${ }^{36}$ Este análisis mostró que, aunque de forma desigual, ha habido avances en cuanto a la gobernanza y gerencia, marco legal, prioridades de investigación en salud, mecanismos de financiamiento y formación de recursos entre los países de la región. Honduras se encontraba entre los países que no poseen estructuras formales de gobernanza ni de gerencia de investigación para la salud. ${ }^{36}$ Partiendo de estos resultados, en 2013 se realizó una revisión sistemática que comparó los métodos de establecimiento de prioridades de investigación para la salud de los países de América Latina y el Caribe. ${ }^{37}$ De igual manera los informes, correspondientes a los países que contaban con documentos que establecían prioridades nacionales de investigación, variaban ampliamente en cuanto a sus objetivos, contenido, difusión y ejecución. Por otra parte, también es necesario promover la formación de talento humano que sea capaz de realizar estudios con diseños experimentales, revisiones sistemáticas, meta-análisis, investigación de la implementación, métodos de planeación de proyectos, entre otros, que generen evidencia óptima para la toma de decisiones, ${ }^{38,39}$ y por consiguiente que contribuya a un mejor sistema de salud pública.

En conclusión, en Honduras aún no se ha logrado establecer un sistema nacional de investigación para la salud, aunque se han dado pasos importantes en esta dirección; por ejemplo, el establecimiento de la Agenda de Investigación para la salud 2015-2018 por la Secretaría de Salud. ${ }^{40}$

Las decisiones a nivel de país deben estar dirigidas a abordar los problemas prioritarios en salud y además basándose en los mejores datos científicos. La ausencia de un sistema nacional de investigación para la salud que responda a las necesidades de la población es ostensible en situaciones como las producidas por la actual pandemia de COVID-19. ${ }^{41}$ Sin embargo, existen plataformas que pueden orientar a los tomadores de decisiones para iniciar este proceso, como por ejemplo EVIPNet (del inglés Evidence-informed Policy Network). Esta iniciativa fue lanzada en 2007 por la OMS como una red innovadora concebida para promover el uso sistemático de resultados de investigaciones en salud de la más alta calidad (evidencias) en la formulación de políticas y toma de decisiones en los países de ingresos bajos y medios. ${ }^{42,43}$ Específicamente el enfoque puede estar orientado a desarrollar el marco legal y regulatorio y la gobernanza del sistema nacional de investigación para la salud en Honduras.

\section{CONTRIBUCIONES}

Ambos autores concibieron, diseñaron y llevaron a cabo el estudio. Ambos contribuyeron a la redacción del artículo, incorporaron las recomendaciones editoriales y aprobaron la versión final.

\section{AGRADECIMIENTOS}

Se reconoce y agradece el apoyo del personal de la Biblioteca Médica Nacional, Facultad de Ciencias Médicas, Universidad Nacional Autónoma de Honduras, Tegucigalpa, en la búsqueda de los artículos. Además, se agradece al Dr. Luis Gabriel Cuervo, Asesor Principal en Investigación en Sistemas de Salud de OPS/OMS, por la revisión crítica del manuscrito. 


\section{REFERENCIAS}

1. Javier Zepeda CA. Reseña Histórica de la Revista Médica Hondureña 19301995. Rev Méd Hondur. 1995;63(4):153-161.

2. Javier Zepeda CA. Reseña Histórica de la Revista Médica Hondureña 19302005. Rev Méd Hondur. 2005;73(Supl 2):4-16.

3. Bu Figueroa E. 90 años de historia. Rev Méd Hondur. 2020;88 (Supl 1):3940.

4. Organización Panamericana de la Salud. Organización Mundial de la Salud. Política de Investigación para la Salud. $49^{a}$ Consejo Directivo, $61^{\text {a }}$ Sesión del Comité Regional CD49/10 (Esp.) [Internet]. Washington D.C.: OPS/OMS; 2009. [citado 2 agosto 2020. Disponible en: https://www.paho.org/hq/images/stories/KBR/Research/politica\%20de\%20investigacion\%20para\%20 la\%20salud.pdf?ua=1

5. World Health Organization. The world health report 2013: research for universal health coverage. Geneva: World Health Organization; 2013.

6. Salicrup LA, Cuervo LG, Cano Jiménez R, Salgado de Snyder N, BecerraPosada F. Advancing health research through research governance. BMJ. 2018;362:k2484.

7. World Health Organization. Global Observatory on Health R\&D. Gross domestic R\&D expenditure on health (health GERD) as a \% of gross domestic product (GDP) [Internet]. Geneva: World Health Organization;2020. [citado 20 Diciembre 2020]. Disponible en: https://www.who.int/research-observatory/indicators/gerd_gdp/en/

8. Hanney SR, Kanya L, Pokhrel S, Jones TH, Boaz A. How to strengthen a health research system: WHO's review, whose literature and who is providing leadership? Health Res Policy Sys. 2020;18:72.

9. Organización Panamericana de la Salud. Organización Mundial de la Salud. Agenda de Salud Sostenible para las Américas 2018-2030: Un Llamado a la Acción para la Salud y el Bienestar en la Región. [Internet]. [citado agosto 2 2020]. Disponible en: https://www.paho.org/es/agenda-salud-sosteniblepara-americas-2018-2030

10. Alger J, F Becerra Posada, A Kennedy, E Martinelli, LG Cuervo, Grupo Colaborativo de la Primera Conferencia Latinoamericana de Investigación e Innovación para la Salud, Rio de Janeiro, Brasil. Sistemas Nacionales de Investigación para la Salud. Sistemas nacionales de investigación para la salud en América Latina: una revisión de 14 países. Rev Panam de Salud Publica. 2009;26(5):447-457.

11. Secretaría de Salud (HN). Agenda de Investigación para la Salud 20152018. .[Internet]. Tegucigalpa: SESAL; 2015. [citado agosto 3, 2020]. Disponible en: http://www.bvs.hn/Honduras/Priorizacion-investigacion-SECSALUD-CRUVAS.pdf

12. Gobierno de la República de Honduras. Ley para la Promoción y Fomento del Desarrollo Científico, Tecnológico y la Innovación. La Gaceta, Decreto 276-2013. Acceso agosto 3 2020. Disponible en: https://www.tsc.gob.hn/ web/leyes/Ley_promo_fomento_desa_cient_2014.pdf

13. Espinoza $E$, Alger J. Ética y conducta responsable en investigación: Una mirada a través de la Revista Médica Hondureña. Rev Méd Hondur. 2020;88(1):33-37.

14. Bermudez A [Editorial]. Investigación Médica en Honduras Paridad Universitaria. Rev Méd Hondur. 1967;35(4):199-200.

15. Membreño A. El Cirujano Clínico Docente: ¿Por qué debe, además de enseñar, escribir? Rev Méd Hondur. 1989;57(1):33-35.

16. Membreño A. La Formación profesional con excelencia académica en los estudios de post grado de la Escuela de Médicina de la UNAH. Rev Méd Hondur. 1989;57(4): 261-263.

17. Membreño A. Análisis Reflexivo, Pragmático y Crítico-Realista sobre la (s) Política (s) de Investigación en la Facultad de Ciencias Médicas de la Universidad Nacional Autónoma de Honduras: los problemas, la realidad y las verdades. Rev Méd Hondur. 1991;59:160-166.

18. Alger J, Castellanos LG. Programa Especial para Investigación y Entrenamiento en Enfermedades Tropicales - TDR. Rev Méd Hondur. 2002;70(2):91-94

19. Alger J. Foro 5, Ginebra, Suiza, Octubre 9-12, 2001 Foro Mundial para la Investigación en Salud. Rev Méd Hondur. 2002;70(1):37-39.
20. Alger J, Quinto Congreso Internacional de Revisión por Pares y Publicación Biomédica. Rev Méd Hondur. 2006;74(1):52-53.

21. Alger J, Espinoza Salvadó I, Valenzuela R, de Haan S, Cuervo LG, Arana $B$, et al. Primera Conferencia Latinoamericana sobre Investigación e Innovación para la Salud, Río de Janeiro, Brasil, abril 15-18, 2008. Rev Méd Hondur. 2008;76(2):88-93.

22. Alger J, Gómez L, Jaramillo A, Saravia NG, Cuervo LG, Halpaap B, Red Inter-Regional de planeación y Evaluación Efectivas de Proyectos de Investigación para la Salud, Cali, Colombia, Abril 2010. Rev Méd Hondur. 2010;78:96-99.

23. Alger J, García MC, Pleitez E, Padilla O, Ramírez L, Sabillón N, et al. Gestión y administración efectiva de revistas científicas: curso y reunión de editores hondureños. Tegucigalpa, Honduras, 10 de Septiembre 2007. Rev Méd Hondur. 2007;75(4):197-200.

24. Sabillon N, Alger J, García MC, Varela C, Varela S, Ramírez L. La Asociación Hondureña de Editores Científicos (AHECI): iniciativa para fortalecer la publicación científica en Honduras. Rev Méd hondur. 2010;78(2):93-95.

25. Sandoval NJ. La Revista Médica Hondureña a la vanguardia de la investigación científica. Rev Méd Hondur. 2011;79 (2):57.

26. Rodriguez Gudiel H. Un nuevo reto en la publicación científica en Médicina. Rev Méd Hondur. 2014;82(1):5.

27. Varela Martínez CE [Editorial]. La Revista Médica Hondureña, la investigación biomédica y las alianzas estratégicas. Rev Méd Hondur. 2002;70(1): 2.

28. Soto RJ, Padgett D, Espinoza Salvadó I. Práctica de la Investigacion en la Facultad de Ciencias Médicas en 1994-1995. Rev Méd Hondur. 1997;65(1):2-8.

29. Fuentes Barahona I, Alger J, Pastrana K. Caracterización de la investigación realizada en el Posgrado de Ginecología y Obstetricia, Facultad de Ciencias Médicas, UNAH, 2012-2015. Rev Med Hondur. 2016;84:6-11.

30. Castejón Cruz OA, Núñez Savoff RA, Bush Wood SW. Características de los trabajos de investigación presentados en los congresos médicos nacionales de Honduras, 2007-2017. Rev Méd Hondur. 2018:86:113-118.

31. Medina MT [Editorial]. Política de investigación en las ciencias biomédicas en Honduras. Rev Méd Hondur. 1996;64(3):83.

32. Villanueva EC, Ribeiro de Abreu D, Cuervo LG, Becerra-Posada F, Reveiz L, ljsselmuiden C. HRWEB: una herramienta para facilitar una mejor gobernanza de la investigación en América Latina y el Caribe. Rev Méd Hondur. 2013;81(1): 46-50.

33. Alger J, Alvarenga RE, Javier CA, Lopez Lutz E, Maradiaga E, Rivera O, et al. Reseña histórica del Congreso Médico Nacional y presentación del XLVI Congreso, Tegucigalpa, 2003. Rev Méd Hondur. 2003;71: 48-54.

34. Varela C, Sánchez J, Joya O, Padgett D, Reyes Ticas A. Conclusiones y recomendaciones del panel de discusión XVI Congreso de los Postgrados de Medicina. Noviembre del 2010. Rev Méd Hondur. 2011;79(1):33-34.

35. Alger J, Rivera O, Maradiaga E, Aguilera EG, Alvarenga RE, Duarte RM, et al. XLVI Congreso Médico Nacional Dr. Pablo J. Cámbar. Rev Méd Hondur. 2003;71(3):156-164.

36. Alger J, Becerra-Posada F, Kennedy A, Martinelli E, Cuervo LG, Grupo Colaborativo de la Primera Conferencia Latinoamericana de Investigación en Innovación para la Salud. Sistemas nacionales de investigación para la salud en América Latina: una revisión de 14 países. Rev Panam Salud Publica. 2009;26(5):447-57.

37. Reveiz L, Elias V, Terry RF, Alger J, Becerra-Posada F. Comparison of national health research priority-setting methods and characteristics in Latin America and the Caribbean, 2002-2012. Rev Panam Salud Publica. 2013;34(1):1-13.

38. Zicker $F$, Cuervo LG, Salicrup LA. Promoting high quality research into priority health needs in Latin America and Caribbean. BMJ. 2018;362:k2492.

39. Fajardo C, Alger, J. Investigación de la implementación: características y oportunidades para su práctica. Rev Méd Hondur. 2019;87(2):85-89.

40. Secretaría de Salud (HN). Agenda de investigación para la salud 2015-2018. Tegucigalpa: SESAL; 2015.

41. Organización Panamericana de la Salud. Organización Mundial de la Salud. 
Informes de la situación de la COVID-19. [Internet]. Washington D.C.: OPS/ OMS; 2020. [citado 22 abril 2020]. Disponible en: https://www.paho.org/es/ informes-situacion-covid-19

42. Alger J, De León MH, Becerra-Posada F. EVIPNet, Red de políticas informadas en la evidencia, una opcion ante la necesidad de traducir la investigacion cientifica en accion en Centro América. Rev Méd Hondur. 2013;81(2):116118.

43. Pantoja T, Barreto J, Panisset U. Improving public health and health systems through evidence informed policy in the Americas. BMJ. 2018;362:k2469.
ABSTRACT. Background: The Revista Médica Hondureña (RMH) registers information about events and evolutionary aspects of clinical assistance, public health, education, and research. Knowledge management for health research organizes information flows and promotes the generation, appropriation, exchange and use knowledge to increase the efficiency of research. Objective: Describe the research management in Honduras through the publications in the RMH (1930-2020). Methods: Scoping review; the search was done under the term research. Articles were selected according to the operational definition of research management based on the objectives of the Health Research policy (PAHO/WHO); they were classified by year of publication, type of article, theme, scope. Results: A total of 24 articles were identified (1989-2020); according to type of article: Opinion 12, Editorial 5, Original 3, Special 2, Bibliographic review 1, History 1 ; according to their general thematic area, articles approached one or more themes, mainly: Research generation 15 , human talent competence 15, research prioritization 9, Diffusion and use of results 9 , strategic alliances 8 . As to its scope, 17 national, 3 regional, and 4 global. Discussion: The RMH reflects progress and challenges of health research in Honduras. It is necessary to promote initiatives and research about governance, practices and standards and evaluation mechanisms of research. The creation of a national system of health research would contribute to promoting the generation and use of scientific evidence of the highest quality and therefore a better public health system in Honduras.

Keywords. Health research policy, Knowledge management for health research, Research, Research promotion. 